\title{
Apparent pulmonary mycetoma following invasive aspergillosis in neutropenic patients
}

\author{
C C KIBBLER, S R MILKINS, A BHAMRA, M A SPITERI, P NOONE, H G PRENTICE \\ From the Departments of Microbiology, Histopathology, Haematology, and Thoracic Medicine, Royal Free \\ Hospital, London
}

\begin{abstract}
After the death from massive haemoptysis of two neutropenic patients who had developed apparent pulmonary mycetomas, two subsequent patients underwent successful resection of similar lesions. Histological examination of these lesions confirmed that these so called mycetomas were masses of devitalised lung tissue infiltrated with fungus. The term mycotic lung sequestrum is therefore proposed to distinguish this condition from a fungus ball arising in a previously formed cavity. A review of 34 similar cases reported previously showed that haemoptysis occurred in about half of the cases and was fatal in just over half of these. Medical treatment appears to have little impact on survival and early consideration of surgical intervention is important.
\end{abstract}

Invasive pulmonary aspergillosis is a major cause of death in neutropenic patients. ' Evidence of it has been found at necropsy in up to $20 \%$ of patients dying with acute leukaemia. ${ }^{2}$ More recently invasive pulmonary aspergillosis has been diagnosed in life in $21 \%$ of patients undergoing treatment for acute leukaemia, and $67 \%$ of these died within two months of diagnosis of the fungal infection. ${ }^{3}$

The range of manifestations of invasive pulmonary aspergillosis has been reported in several large series of cases. ${ }^{45}$ The most common clinical and histological manifestations were necrotising bronchopneumonia and haemorrhagic pulmonary infarction, occurring in about $60 \%$ of cases. Recently, however, apparent mycetoma formation (seen on radiographs as an air crescent surrounding a dense opacity but differing histologically from the classical mycetoma occurring in patients with pre-existing lung disease) has been increasingly described. Since the aetiology and management of this syndrome is controversial we describe our experience with four such patients and review 34 published cases in an attempt to resolve these issues.

\section{Patients and methods}

Nine cases of invasive pulmonary aspergillosis have been diagnosed before death among patients undergo-

Address for reprint requests: Dr C C Kibbler, Department of Clinical Microbiology, University College Hospital, London WCIE 6AU.

Accepted 18 November 1987 ing treatment in the haematology unit in the past 18 months. Two of the nine patients had radiological evidence of apparent mycetoma formation. The management of these two patients was influenced by the clinical course of two similar patients who had both died from massive haemoptysis. Brief case reports of all four patients are presented.

We reviewed the available publications on invasive pulmonary aspergillosis occurring in neutropenic patients and progressing to mycetoma formation. ${ }^{35-14}$ From this we extracted the following data: underlying disease, date of onset and duration of neutropenia, surgical intervention and outcome, haemoptysis (life threatening or otherwise), antifungal treatment, serological evidence of aspergillus infection, and duration of survival. Cases appearing in more than one publication were included once only. Some reports that described patients with apparent pulmonary mycetomas gave insufficient detail to enable the cases to be included in this survey. ${ }^{415}$

\section{Case reports}

Details of the four patients are shown in table 1. All patients had a normal chest radiograph before the onset of neutropenia (neutrophils $<1.0 \times 10^{9} / 1$ ), ? which in the first two cases followed chemotherapy and total body irradiation as conditioning for allogeneic bone marrow transplantation. The other two patients became neutropenic after induction treatment $\stackrel{\AA}{\AA}$ for acute leukaemia. Localised shadowing appeared on the chest radiograph in three patients after the 
Table 1 Details of the 38 patients and infecting Aspergillus organisms*

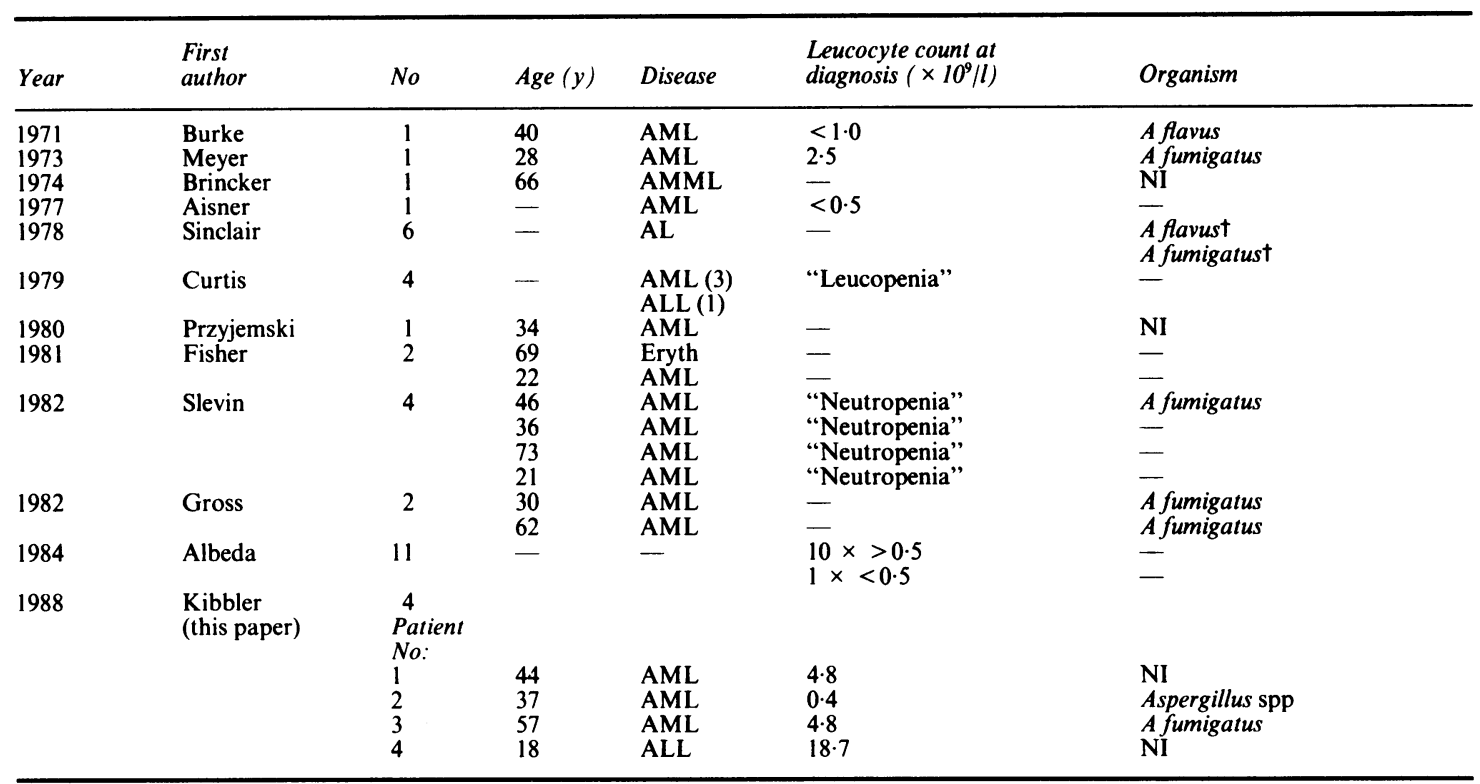

"Data are given only where specific patients or patient numbers have been documented.

tTwo patients.

AML, acute myeloid leukaemia; ALL, acute lymphoblastic leukaemia; AL acute leukaemia; AMML, acute myelomonocytic leukaemia; Eryth, erythroleukaemia; NI, not isolated on culture. A dash means not discussed.

onset of neutropenia. Patient 4 was febrile for seven days but did not develop radiological evidence of infection until six days after resolution of neutropenia. Three of the four patients became afebrile after starting treatment with broad spectrum antibiotics, which initially suggested a bacterial pneumonia; and indeed in patient 3 Klebsiella pneumoniae was grown from bronchoalveolar lavage fluid and blood cultures at the onset of radiological shadowing. All four patients underwent fibreoptic bronchoscopy and lavage but, apart from the Klebsiella obtained from patient 3 , only normal upper respiratory tract organisms were isolated, despite prolonged incubation of Sabouraud plates at $20^{\circ}, 30^{\circ}$, and $37^{\circ} \mathrm{C}$ in an attempt to culture fungi.

The radiological shadowing progressed in all patients until a single cavity appeared containing a rounded opacity (the "air crescent sign"-fig 1). Once this had occurred invasive fungal disease was suspected and treatment was started with intravenous amphotericin B.

The lesions in patients 1 and 2 persisted and both died of massive haemoptysis. A policy of early surgical intervention in future cases was therefore formulated and, as a result, resection of the affected lobe was performed 15 days after diagnosis in patient 3 and eight days in patient 4.

Patient 3 developed atrial fibrillation and an episode of pulmonary oedema after resection, but both patients were discharged well 41 days after surgery. They subsequently died in a leukaemic relapse.

Gross examination of the resected or post mortem tissues in each case showed areas suggestive of necrosis, which had a tendency to separate from the

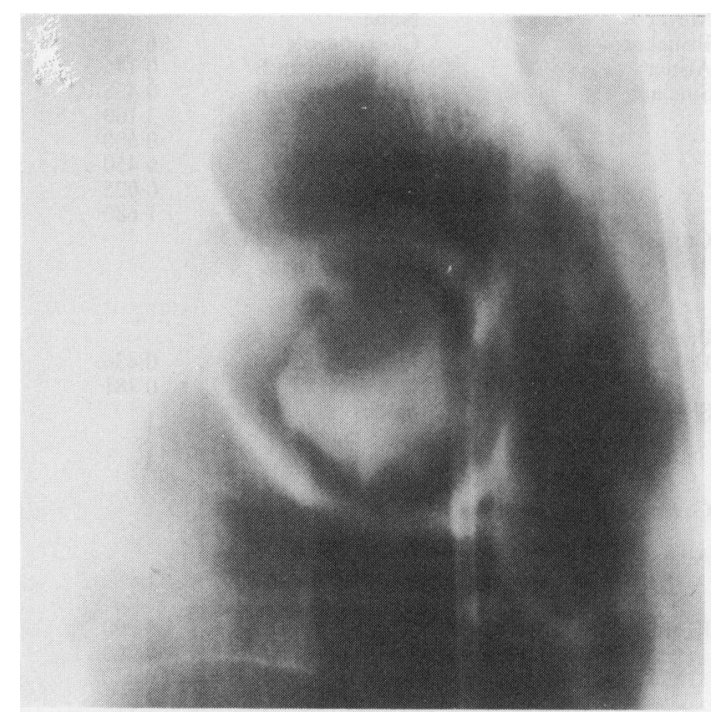

Fig 1 Tomogram of right upper lobe lesion in case 3, showing the air crescent sign. 
110

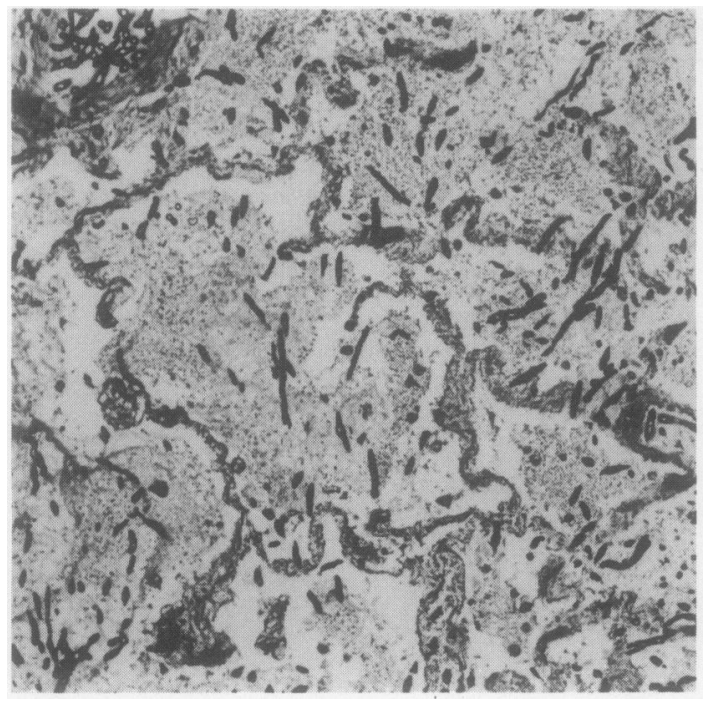

Fig 2 Fungal hyphae permeating sequestrum of lung tissue in case 3. (Grocott stain.)

adjacent lung tissue. Infarction was confirmed histologically and dichotomously branched septate hyphae were present within the infarcted areas and adjacent tissue (fig 2). There was considerable fungal
Kibbler, Milkins, Bhamra, Spiteri, Noone, Prentice invasion of thrombosed blood vessels. The firm edges of the "cavities" seen macroscopically were due to granulation tissue and fibrosis in the viable lung tissue. None of the specimens showed evidence of previous disease, such as tuberculosis.

Culture of the affected lung tissue yielded Aspergillus fumigatus in patient 3 and an unidentified aspergillus species in patient 2 .

\section{Results}

Data on 38 patients, including the four described in this paper, are shown in tables 1 and 2. Where iv information was available the most common underlying disease was acute myeloblastic leukaemia (17 out of 21 cases). Almost all the patients were neutropenic when shadowing was first noted on the chest radiograph. The subsequent neutrophil counts were studied in detail only by Albeda et al, ${ }^{3}$ who reported radiological evidence of mycetoma on average two days after the count exceeded $0.5 \times 10^{9} / 1$. In our series the lesion was diagnosed an average of 16 days after a count of more than $1.0 \times 10^{9} / 1$. In reports where it is discussed the appearance of cavitation on the chest radiograph seems to have followed bone marrow recovery in most cases, though Slevin et al ${ }^{13}$ describe one case where haematological remission was not

Table 2 Treatment, incidence of haemoptysis, and survival

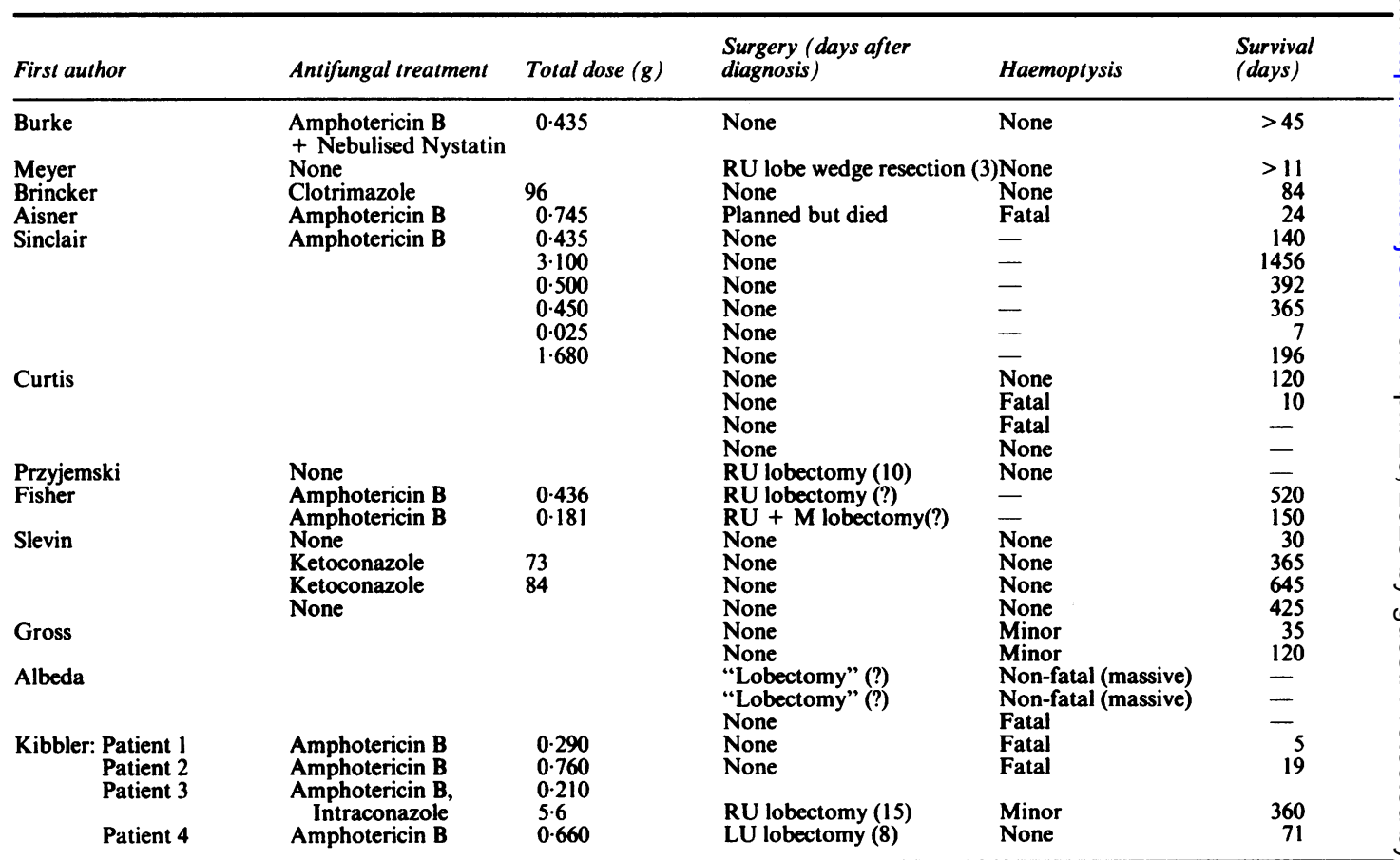

RU, right upper; $L U$, left upper; $M$, middle. A dash means not discussed. 
achieved and there are three cases in table 1 where lesions developed before the neutrophil count exceeded $0.5 \times 10^{9} / 1$.

Haemoptysis occurred in 11 out of 22 patients, six of whom died as a direct result. The mean time interval between radiological diagnosis of cavitation and haemoptysis was 7 (range 1-24) days.

The platelet count at the time of haemoptysis was known for six of the 11 patients in whom haemoptysis occurred and in each case was at least $26 \times 10^{9} / 1$. The absolute platelet count can be determined for three cases, of which one was in the normal range $(231 \times$ $\left.10^{9} / 1\right)$. The clotting profiles were said to be normal in the three patients with massive haemoptysis in Albeda's series. ${ }^{3}$ In our three patients with haemoptysis the prothrombin and partial thromboplastin times were within the normal range.

Surgery was undertaken in eight patients, including three who had massive haemoptysis. Although morbidity related to surgery was not examined in any of the series, no deaths due to surgery were documented. The mean duration of survival in the four patients undergoing surgery for whom data are available was 305 (SD 177) days, compared with 246 (SD 344) days in the 18 patients treated conservatively.

Information on the infecting organism was available for only 10 patients. Seven cases were due to Aspergillus fumigatus, three to A flavus, and one to an unidentified species of Aspergillus. Antibodies against Aspergillus species antigens were measured by various methods (aspergillus precipitins ${ }^{570-12}$ and immunoblot analysis ${ }^{16}$ ), in only 10 patients and were detectable in 7 .

Details of treatment where specified are shown in table 2. There was no significant difference in duration of survival between patients given no effective antifungal therapy (mean survival 296 (SD 214) days) and those given a total dose of up to $2.0 \mathrm{~g}$ amphotericin (mean survival 208 (174) days).

\section{Discussion}

Attempts to examine management strategies in invasive pulmonary aspergillosis are fraught with difficulties. There is a wide range of disease and the immune status of the affected patients varies enormously. We have restricted our study to one specific presentation of the disease and the conclusions drawn cannot be extrapolated to other forms.

Our findings confirm that pulmonary "mycetomas" occurring in neutropenic patients with previously normal lungs consist of necrotic pulmonary tissue infiltrated with fungus. ${ }^{1111314}$ Thus the term "mycetoma," implying a "fungal ball" arising within a previously formed cavity, is a misnomer. Several authors have referred to the concept of a "seques- trum" of devitalised lung tissue $e^{10114}$ and we propose the term mycotic lung sequestrum to describe such a lesion.

Invasive aspergillosis characteristically affects the blood vessels, causing thrombosis and local infarction. Invasion of previously infarcted lung tissue by saprophytic fungi, including Aspergillus species, is well recognised $^{17}$ and invasion of the lung by Aspergillus could have been the consequence rather than the cause of infarction in the cases in this survey. Such a sequence of events is difficult to substantiate, however, in neutropenic patients, and pulmonary infarction in the absence of invasive fungal infection appears to be extremely unusual. This suggests that thrombosis and subsequent pulmonary infarction follow invasion with the fungus, rather like the progressive rhinocerebral aspergillosis seen in such patients. It is not clear why some patients develop necrotising bronchopneumonia and haemorrhagic pulmonary infarction, while others have localised disease with subsequent sequestrum formation, though invasion of particular vessels may play a part in determining the extent and pattern of necrosis. The mechanism by which the sequestrum becomes separated from surrounding normal lung might depend on neutrophil proteolytic enzyme activity as previously suggested, ${ }^{3}$ as most cases have occurred after bone marrow recovery. There are reports of cases where this did not occur, and not all patients with invasive pulmonary aspergillosis whose bone marrows regenerate form a mycotic lung sequestrum. Why certain patients form a cavity without a sequestrum is not clear. Perhaps sufficient central fungal infiltration is required to prevent resorption of the mass.

The radiological appearances suggesting mycotic lung sequestrum are not specific and the differential diagnosis has to take account of tuberculosis, nocardiasis, lung abscess due to other bacteria, hydatid disease, cysticercosis, and neoplasm..$^{30}$ Mucor species may also cause infection. ${ }^{18}$ There are limited data on methods of diagnosis in these patients. Fibreoptic bronchoscopy yielded the diagnosis in a third of patients and antibody was detected in seven out of 10 . The radiological features may be the best guide to diagnosis in most patients. The organism was grown from tissue either before or after death in only half the cases, suggesting that, even with bronchoscopy, attempts at a diagnosis based on culture alone are likely to be inadequate. Failure to culture the organism may have been due to prior treatment with antifungal agents in some cases, but Aspergillus could not be cultured from $30 \%$ of tissue specimens in a series where most patients were untreated. ${ }^{4}$

This review shows that haemoptysis is life threatening in this condition and in most cases occurs within one week of diagnosis. There is no way of predicting 
which patients are at risk, thought premonitory haemoptysis occurs in a small number.

Once the patient has survived the initial risk period the best guide to prognosis would appear to be the state of the bone marrow. There were at least five cases in which spontaneous resolution occurred without effective treatment. Unfortunately, the patients who die during the initial period usually do so when in remission and hence would otherwise have had a relatively good prognosis. Since there was no evidence that antifungal treatment improved survival we recommend surgical intervention when possible. The results of the survey suggest that resection should take place as soon as possible after diagnosis, preferably within the first week. Surgery was not associated with major problems in the eight patients in whom it was carried out, presumably because they were no longer appreciably neutropenic or thrombocytopenic and were relatively young and fit, apart from their underlying disease. A survey of published cases is of necessity incomplete and there may well be unpublished cases of mycotic lung sequestrum in which surgery has been associated with death. Surgery in patients with aspergilloma has been estimated to carry a mortality of $8 \%{ }^{19}$; but these patients are usually older, with underlying lung disease.

In conclusion, we believe that a neutropenic patient developing pneumonia should receive intravenous amphotericin B (1.0-1.5 mg/ $\mathrm{kg}$ a day) in the absence of a response to broad spectrum antibiotics while serological investigation and, if possible, fibreoptic bronchoscopy with bronchoalveolar lavage is undertaken. The patient must be carefully monitored with frequent chest radiographs, particularly after marrow regeneration. At the first suspicion of cavitation tomography should be undertaken, and if this shows the appearances of mycotic lung sequestrum formation a surgical opinion is advised.

We are grateful to Dr James Burnie, who kindly performed the immunoblot analyses for aspergillus antibodies on sera from our patients; and we would like to thank Professor AV Hoffbrand for permission to report two of his patients, Mrs Megan Evans for preparing the manuscript, and $\mathrm{Mr} \mathrm{F}$ Moll for the photography.

\section{References}

1 Williams DM, Krick JA, Remington JS. Pulmonary infection in the compromised host. Am Rev Respir Dis
1976;114:359-4.

2 Degregorio MW, Lee WMF, Linker CA, Jacobs HA, Ries CA. Fungal infections in patients with acute leukemia. Am J Med 1982;73:543-8.

3 Albeda SM, Talbot GH, Gerson SL, Miller WT, Cassileth PA. Pulmonary cavitation and massive hemoptysis in invasive pulmonary aspergillosis. Am Rev Respir Dis 1985;131:115-20.

4 Young RC, Bennett JE, Vogel CI, Carbone PP, DeVita VT. Aspergillosis: the spectrum of the disease in 98 patients. Medicine (Baltimore) 1970;49:147-73.

5 Meyer RD, Young LS, Armstrong D, Yu B. Aspergillosis complicating neoplastic disease. Am J Med 1973;54: 6-15.

6 Burke PS, Coltman CA. Multiple pulmonary aspergillomas in acute leukemia. Cancer 1971;28:1289-92.

7 Brincker H, Hansen PB. Development of pulmonary aspergillomas coinciding with induction of remission in acute myeloid leukaemia. Dan Med Bull 1974;21: $37-40$.

8 Aisner J, Schimpff SC, Wiernik PH. Treatment of invasive aspergillosis: relation of early diagnosis and treatment to response. Ann Intern Med 1977;86:539-43.

9 Sinclair AJ, Rossof AH, Coltman CA. Recognition and $\vec{\oplus}$ successful management in pulmonary aspergillosis in $\infty$ leukemia. Cancer 1978;42:2019-24.

10 Curtis AMcB, Walker Smith GJ, Ravin CE. Air crescent sign of invasive aspergillosis. Radiology 1979;133: 17-21.

11 Przyjemski C, Mattii R. The formation of pulmonary mycetomata. Cancer 1980;46:1701-4.

12 Fisher RD, Armstrong D, Yu B, Gold JWM. Invasive aspergillosis: progress in early diagnosis and treatment. Am J Med 1981;71:571-7.

13 Slevin ML, Knowles GK, Phillips MJ, Stansfield AG, Lister TA. The air crescent sign of invasive pulmonary aspergillosis in acute leukaemia. Thorax 1982;37: $554-5$.

14 Gross HH, Spitz HB, Felson B. The mural nodule in cavitary opportunistic pulmonary aspergillosis. Radiology 1982;143:619-33.

15 Spearing RL, Pamphilon DH, Prentice, AG. Pulmonary aspergillus in immunosuppressed patients with haematological malignancies. $Q J$ Med 1986;230: 611-25.

16 Matthews R, Burnie JP, Fox A, Tabaqchali S. Immunoblot analysis of serological responses in invasive aspergillosis. J Clin Pathol 1985;38:1300-3.

17 Buchanan DR, Lamb D. Saphrophytic invasion of infarcted pulmonary tissue by Aspergillus species. Thorax 1982;37:760-4.

18 Funada $H$, Misawa T, Nakao S, Saga T, Hattori K-I. The air crescent sign of invasive pulmonary mucormycosis in acute leukemia. Cancer 1984;53:2721-3.

19 Glimp RA, Bayer AS. Pulmonary aspergilloma: diagnostic and therapeutic considerations. Arch Intern Med 1983;143:303-8. 